\title{
Maxima of Gamma random variables and other Weibull-like distributions and the Lambert $W$ function
}

\author{
Armengol Gasull • José A. \\ López-Salcedo • Frederic Utzet
}

Received: date / Accepted: date

\begin{abstract}
In some applied problems of signal processing the maximum of a sample of $\chi^{2}(m)$ random variables is computed and compared with a threshold to assess certain properties. It is well known that this maximum, conveniently normalized, converges in law to a Gumbel random variable; however, numerical and simulation studies show that the norming constants that are usually suggested are inaccurate for moderate or even large sample sizes. In this paper we propose, for Gamma laws (in particular, for a $\chi^{2}(m)$ law) and other Weibull-like distributions, other norming constants computed with the asymptotics of the Lambert $W$ function that significantly improve the accuracy of the approximation to the Gumbel law.
\end{abstract}

Keywords Weibull-like distributions - Gamma distributions · Extreme value theory $\cdot$ Lambert function

Mathematics Subject Classification (2000) 60G70 - 60F05 • 62G32 . $41 \mathrm{~A} 60$

\section{Introduction}

The origin of this paper is to be found in the authors' attempt to apply Extreme Value Theory to the maxima of $\chi^{2}$ random variables in a problem of signal processing (see Turunen [12]). The goal was to characterize accurately the detection performance of a Global Navigation Satellite System (GNSS)

A. Gasull and F. Utzet

Department of Mathematics, Facultat de Ciències, Universitat Autònoma de Barcelona, 08193 Bellaterra, Barcelona, Spain.

E-mail: gasull@mat.uab.cat, utzet@mat.uab.cat

J. A. López-Salcedo

Department of Telecommunications and Systems Engineering, Engineering School, Universitat Autònoma de Barcelona, 08193 Bellaterra, Barcelona, Spain.

E-mail: jose.salcedo@uab.cat 
receiver, whose main task is to provide positioning information by processing the signals emitted from Earth-orbiting satellites (see Seco-Granados et al. [11]). In practice (see Online Appendix 1) this parallel acquisition approach normally involves computing the maximum of 10 to $10^{6} \chi^{2}(\mathrm{~m})$ random variables, for $m$ typically up to 20, as happens in demanding applications such as indoor positioning or space navigation. In this context, it is interesting to note that $\chi^{2}$ random variables are in the domain of attraction for maxima of the Gumbel law. However, the norming constants that are usually proposed give inaccurate results for such sample sizes (see Subsection 5.3). Then we realized that the computations needed to obtain these constants were related to the Lambert $W$ function (see Corless et al. [2]), and that the asymptotic expansion for that function helps to improve the norming constants very much.

We show that the centering constant for the maxima of $n$ independent and identically distributed (i.i.d.) random variables with a distribution that generalizes Weibull distribution can be expressed in closed form in terms of the negative branch of a real Lambert $W$ function. The asymptotic expansion of this function is well known, and the centering constant that is deduced using standard methods of asymptotic analysis corresponds to the two dominant terms of that expansion, loosely speaking, of the form $C_{1} \log n+C_{2} \log \log n$. However, for typical sample sizes, the results are quite inaccurate, and we propose to add one more term of that asymptotic expansion, of the form $C_{3} \log \log n / \log n$; this term goes to zero when $n \rightarrow \infty$, but so slowly that it cannot be neglected. This approach is also applied to a family of distributions called Weibull-like by Embrechts et al. [4, p. 155] (see Section 2) that includes Gamma laws (in particular $\chi^{2}$ laws), to which we pay special attention. In this latter case, we need a double enhancement of the standard technique: on the one hand, the usual distribution tail equivalent to a Gamma distribution needs to be improved; on the other hand, using the asymptotic expansion of a generalization of Lambert $W$ function, we add an additional term, that, as before, goes to zero when the sample size increases, but also very much helps to get accurate approximations.

The contents of the paper are the following. In Section 2 we introduce the class of generalized Weibull distributions and its tail-equivalent distributions, called Weibull-like distributions; we also recall some essential facts about Extreme Value Theory. In Section 3 we study a particular simple case of a generalized Weibull distribution and we describe the problem of the inaccuracy of the norming constants; also we introduce the Lambert $W$ function and its asymptotics. In Section 4 we study the velocity of convergence of the maxima of generalized Weibull laws and show the importance of the election of the norming constants; we also introduce a generalization of Lambert $W$ function and its asymptotic expansion. In Section 5 we apply these results to the maxima of Gamma laws. Finally, an example with real data is sketched in Section 6 , and given in full detail in Online Appendix 1 (see Supplementary material). Some technical matters are placed in Online Appendix 2. 


\section{Weibull-like distributions and their maxima}

To introduce notation and to describe the context of the paper we recall a few basic facts from Extreme Value Theory. Let $X_{1}, \ldots, X_{n}$ be i.i.d. random variables with common cumulative distribution function $F$, and denote by $M_{n}$ its maximum,

$$
M_{n}=\max \left\{X_{1}, \ldots, X_{n}\right\} .
$$

It is said that $F$ is in the domain of attraction for maxima of the Gumbel law if there are sequences of real numbers $\left\{a_{n}, n \geq 1\right\}$ and $\left\{b_{n}, n \geq 1\right\}$ (the norming -or normalizing- constants) with $a_{n}>0$ such that

$$
\lim _{n}\left(M_{n}-b_{n}\right) / a_{n}=H, \text { in distribution, }
$$

where $H$ is a Gumbel random variable, with distribution function

$$
\Lambda(x)=\exp \left\{-e^{-x}\right\}, x \in \mathbb{R}
$$

The norming constants can be taken (see, for example, Resnick [8, Prop. 1.11])

$$
b_{n}=F^{-1}\left(1-n^{-1}\right)
$$

and

$$
a_{n}=A\left(b_{n}\right),
$$

where $A(x)$ is an auxiliary function of the distribution function $F$. Auxiliary functions are not unique though they are asymptotically equal. However, under certain conditions (in particular, $F$ should have density, denoted by $f$, for $x>x_{0}$, for some $x_{0}$ ) an auxiliary function is (see again Resnick [8, Prop. 1.11])

$$
A(x)=(1-F(x)) / f(x) .
$$

We remark that from the standard proof of the convergence (1) it is not deduced that these constants produce more accurate results than other constants computed with other auxiliary functions or other ways.

In general, equation (3) has no closed solution, and then, to obtain explicit expressions of $b_{n}$ and $a_{n}$, the following two properties are used. The first one can be called simplification by tail equivalence (see Resnick [8, Prop. 1.19]). The second one is a property of the convergence in law applied to this context.

Property 1 Let $F$ be a distribution function in the domain of attraction of a Gumbel law, and let $G$ be another distribution function right tail equivalent to $F$,

$$
\lim _{x \rightarrow \infty}(1-G(x)) /(1-F(x))=1 .
$$

Then $G$ is also in the domain of attraction of the Gumbel law and the norming constants of $F$ and $G$ can be taken equal. 
Property 2 Let $F$ be a distribution function that belongs to the domain of attraction for maxima of the Gumbel law, with norming constants $\left\{a_{n}, n \geq 1\right\}$ and $\left\{b_{n}, n \geq 1\right\}$. If the sequences $\left\{\widetilde{a}_{n}, n \geq 1\right\}$ and $\left\{\widetilde{b}_{n}, n \geq 1\right\}$ satisfy

$$
\lim _{n} a_{n} / \widetilde{a}_{n}=1 \text { and } \lim _{n}\left(b_{n}-\widetilde{b}_{n}\right) / a_{n}=0,
$$

then $\left\{\widetilde{a}_{n}, n \geq 1\right\}$ and $\left\{\widetilde{b}_{n}, n \geq 1\right\}$ are also norming constants for $F$.

Our main objective is to study the maxima of distributions called Weibulllike distributions by Embrechts et al. [4, p. 155] which are right tail equivalent to an extension of Weibull distribution. For convenience of the exposition, we first introduce a new family of probability distributions that are intermediate between the Weibull and Weibull-like distributions, and they have the important advantage that its quantile function $F^{-1}$ can be written explicitly in terms of the Lambert $W$ function, which is very well known (see Corless et al. [2]). Therefore the norming constants can be efficiently computed since Lambert $W$ function is efficiently implemented in algebra computer systems. Specifically, in this paper a probability distribution function $F$ of the form

$$
F(x)=1-K x^{\alpha} \exp \left\{-C x^{\tau}\right\}, x \geq x_{0},
$$

for some $x_{0}$, where $K, C, \tau>0$, and $\alpha \in \mathbb{R}$, will be called a generalized Weibull distribution. The standard Weibull law $W(\lambda, \nu)$, where $\lambda>0$ is the scale parameter and $\nu>0$ the shape parameter, corresponds to the case $\alpha=0, \tau=\nu, C=1 / \lambda^{\nu}, K=1$ and $x_{0}=0$; in particular an exponential law of parameter $\lambda>0$ has $\alpha=0, \tau=1$ and $C=\lambda$, and a $\chi^{2}(2)$ law has $\alpha=0, \tau=1, C=1 / 2$. The distribution function (6) is a Von Mises function since it has the representation $1-F(x)=C_{0} \exp \left\{-\int_{x_{0}}^{x}(1 / g(u)) d u\right\}$, for $x \geq x_{0}$, where $g(u)=\left(C \tau u^{\tau-1}-\alpha / u\right)^{-1}$. This implies that generalized Weibull distributions belong to the domain of attraction for maxima to the Gumbel law (Resnick [8, Prop. 1.4]).

In agreement with Embrechts et al. [4, p. 155], a probability distribution function that is right tail equivalent to a generalized Weibull distribution is said to be a Weibull-like distribution. A main example is the Gamma law $G(\nu, \theta)$ (with $\alpha=\nu-1, \tau=1$ ), and, in particular, the $\chi^{2}(m)$ law, see Section 5. The normal law is also Weibull-like with $\alpha=-1$ and $\tau=2$; however, this case has special properties: on the one hand, there is a remarkable result of Hall [5] where the velocity of convergence of normal extremes is computed; on the other hand, the fact that $\alpha<0$ introduces important changes in our approach that we comment in Subsection 4.4. Given that we are mainly interested in the Gamma law we will assume from now on that $\alpha>0$ and $\tau \geq 1$.

Let $X_{1}, \ldots, X_{n}$ be i.i.d. random variables with Weibull-like distribution function $G$, right tail equivalent to a generalized Weibull distribution function $F$ of the form (6). Thanks to Property 1, the norming constants can be taken

$$
b_{n}=F^{-1}\left(1-n^{-1}\right) \quad \text { and } \quad a_{n}=\left(C \tau b_{n}^{\tau-1}-\alpha / b_{n}\right)^{-1},
$$


where for the expression of $a_{n}$ we have used the auxiliary function (5) associated to $F$. From that expressions, by using Property 1 and asymptotic analysis, there are deduced explicit expressions of the norming constants, as the following ones given by Embrechts et al. [4, p. 155],

$$
\begin{aligned}
& b_{n}^{\prime}=\left(C^{-1} \log n\right)^{1 / \tau}+\frac{1}{\tau}\left(C^{-1} \log n\right)^{1 / \tau-1}\left(\frac{\alpha}{C \tau} \log \left(C^{-1} \log n\right)+\frac{1}{C} \log K\right), \\
& a_{n}^{\prime}=(C \tau)^{-1}\left(C^{-1} \log n\right)^{1 / \tau-1} .
\end{aligned}
$$

These will be called the standard constants. Our purpose is to show that, for moderate or even quite large sample sizes, the election of the norming constants plays a major role in the velocity of convergence of (1), and that it is possible to choose other constants that produce more accurate results than the standard ones.

\section{The simplest case}

To illustrate the inaccuracy of the standard constants and the techniques that we use, we first consider the following particular case of a generalized Weibull distribution:

$$
F(x)=1-e x e^{-x}, x \geq 1,
$$

where $K=e$ and $C=\tau=\alpha=1$. We see in Remark 3 at the end of Subsection 5.2 that the study of the properties of the norming constants of a Gamma law with shape parameter equal to 2 can be reduced to this case.

The standard constants (8) corresponding to the distribution function (9) are

$$
b_{n}^{\prime}=\log n+\log \log n+1 \quad \text { and } \quad a_{n}^{\prime}=1 .
$$

Consider a sample size $n=100$. From the first equation in (7) and (14) below, we get $b_{n} \approx 7.6384$ (see Subsections 3.1 and 3.2), and from the second equation in (7) we obtain $a_{n} \approx 1.1506$. The standard constants $(10)$ are $b_{n}^{\prime} \approx 7.1323$ and $a_{n}^{\prime}=1$. In Figure 1 there is a plot of the density of the Gumbel law

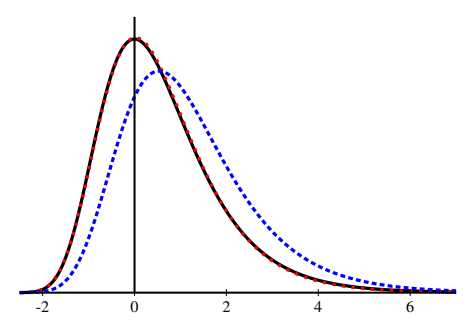

Fig. 1: Solid line: Gumbel density. Red dotted line: Density of $Y_{n}$. Blue dashed line: Density of $Y_{n}^{\prime}$. 
and the densities of the random variables $Y_{n}=\left(M_{n}-b_{n}\right) / a_{n}$ and $Y_{n}^{\prime}=$ $\left(M_{n}-b_{n}^{\prime}\right) / a_{n}^{\prime}$. Observe that the densities of the Gumbel law and of $Y_{n}$ are practically indistinguishable; in contrast, the density of $Y_{n}^{\prime}$ is quite far from the Gumbel density. As a consequence, this plot illustrates that for $n=100$, the distribution of $Y_{n}$ is very near to the limit, but $Y_{n}^{\prime}$ is not, so the approximate norming constants are very important for such a sample size (and much bigger sample sizes, see Table 1). Then we try to improve the accuracy of $Y_{n}^{\prime}$ choosing other norming constants. To get some insight in this question we study the first equation in (7) using the Lambert $W$ function.

\subsection{The Lambert $W$ function}

In the real case the Lambert function $W$ is defined implicitly through the real solution of the equation

$$
W(x) e^{W(x)}=x .
$$

Equivalently, $W$ is the inverse of the function $f(t)=t e^{t}$. The Lambert function has two branches (see Figure 2), the principal one, denoted by $W_{0}$, is defined on $[-1 / e, \infty)$, satisfying $-1 \leq W_{0}(x)$, and a secondary one, called the negative branch, denoted by $W_{-1}$, defined on $[-1 / e, 0)$ satisfying $W_{-1}(x) \leq-1$ (in fact, $\left.W_{0}(-1 / e)=W_{-1}(-1 / e)=-1\right)$ and $\lim _{x \rightarrow 0^{-}} W_{-1}(x)=-\infty$.

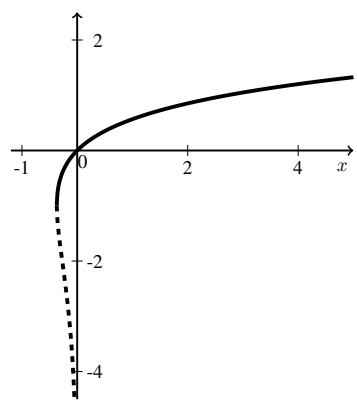

Fig. 2: Solid line: Principal branch of the real Lambert $W$ function. Dashed line: negative branch

We are interested in the negative branch; its asymptotic expansion is (Corless et al. [2, pp. 349-350], see also De Bruijn [3, pp. 25-27]), for $x \rightarrow 0^{-}$,

$W_{-1}(x)=L_{1}(-x)-L_{2}(-x)+\frac{L_{2}(-x)}{L_{1}(-x)}+\frac{L_{2}(-x)\left(-2+L_{2}(-x)\right)}{2 L_{1}^{2}(-x)}+O\left(\frac{L_{2}^{3}(-x)}{L_{1}^{3}(-x)}\right)$,

where

$$
L_{1}(x)=\log x \quad \text { and } \quad L_{2}(x)=\log |\log x| .
$$


Notations As usual, we write that $g(x)=O(h(x))$ when $x \rightarrow \infty$ if there is a point $x_{0}$ and a constant $C$ such that $|g(x)| \leq C h(x)$, for all $x>x_{0}$. We say that $g(x)$ and $f(x)$ are asymptotically equal and write $h \sim g$ if $\lim _{x \rightarrow \infty} g(x) / h(x)=$ 1. Similar notations are used when we consider $x \rightarrow a$.

Remark 1 All the explicit computations related to the Lambert $W$ function have been done with the function lambertW of the package emdbook of the software $\mathbf{R}$.

3.2 Computation of the norming constants via Lambert function

To compute the norming constants, the first equation in (7) for $F$ given in (9) is $e b_{n} e^{-b_{n}}=1 / n$, and hence,

$$
b_{n}=-W(-1 /(e n)) .
$$

By construction $\lim _{n} b_{n}=\lim _{n} F^{-1}\left(1-n^{-1}\right)=\infty$, so in (13) it is needed to consider the negative branch:

$$
b_{n}=-W_{-1}(-1 /(e n)) .
$$

The asymptotic behaviour of $b_{n}$ can be deduced from (11) and gives

$$
b_{n}=\log n+1+\log (\log n+1)+\log (\log n+1) /(\log n+1)+\cdots .
$$

From the second equation in (7) we deduce

$$
a_{n}=b_{n} /\left(b_{n}-1\right) \text {. }
$$

\subsection{Comparison of the velocity of convergence}

The convergence (1) is equivalent to that for every $x \in \mathbb{R}, \lim _{n} F^{n}\left(a_{n} x+b_{n}\right)=$ $\Lambda(x)$, where $\Lambda(x)$ is given in (2). We prove in Theorem 1 that

$$
F^{n}\left(a_{n} x+b_{n}\right)-\Lambda(x)=g(x) / \log ^{2} n+O\left(\log \log n / \log ^{2} n\right) .
$$

Moreover, under some conditions, for any set of admissible constants $\widetilde{a}_{n}, \widetilde{b}_{n}$,

$$
F^{n}\left(\widetilde{a}_{n} x+\widetilde{b}_{n}\right)-\Lambda(x)=h(x)\left(b_{n}-\widetilde{b}_{n}\right)+O(1 / \log n),
$$

where the functions $g$ and $h$ are bounded. In particular, for the standard norming constants $(10), b_{n}-b_{n}^{\prime} \sim \log \log n / \log n$. Then, taking $\widetilde{a}_{n}=a_{n}^{\prime}$ and $\widetilde{b}_{n}=b_{n}^{\prime}$, the velocity of convergence of the normalized maxima to the Gumbel law becomes of that order. However, if we take one more term of the asymptotic expansion of Lambert $W$ function in agreement with (15),

$$
b_{n}^{\prime \prime}=\log n+1+\log (\log n+1)+\log (\log n+1) /(\log n+1),
$$


then $b_{n}-b_{n}^{\prime \prime} \sim 0.5(\log \log n / \log n)^{2}$. Moreover, if $a_{n}^{\prime \prime}=b_{n}^{\prime \prime} /\left(b_{n}^{\prime \prime}-1\right)$ (that is, using the auxiliary function (5)), by Remark 2 of Theorem 1, the order of convergence to the Gumbel law improves until order $(\log \log n / \log n)^{2}$. Thus, from a practical point of view, there is almost no difference between the use of the pair $a_{n}, b_{n}$ or $a_{n}^{\prime \prime}, b_{n}^{\prime \prime}$. In Table 1 it is illustrated that the value of the constant $b_{n}^{\prime \prime}$ approximates $b_{n}$ much better than $b_{n}^{\prime}$, specially for moderate sample sizes, and, as we commented, this has a strong repercussion on the velocity of convergence of the normalized maxima. Of course, we could add more terms to $b_{n}^{\prime \prime}$, but Table 1 and the fact that taking $b_{n}$ or $b_{n}^{\prime \prime}$ the order of convergence to the Gumbel law is very similar, suggest that it is unnecessary.

\begin{tabular}{rcrrrrc}
\hline $\boldsymbol{n}$ & 10 & $10^{2}$ & \multicolumn{1}{c}{$10^{3}$} & $10^{4}$ & $10^{5}$ & $10^{6}$ \\
$b_{n}$ & 4.8897 & 7.6384 & 10.2334 & 12.7564 & 15.2366 & 17.6884 \\
$b_{n}^{\prime}$ & 4.1366 & 7.1323 & 9.8404 & 12.4307 & 14.9564 & 17.4413 \\
$b_{n}^{\prime \prime}$ & 4.8590 & 7.6364 & 10.2371 & 12.7613 & 15.2416 & 17.6931 \\
\hline
\end{tabular}

Table 1: Comparison of the constants for the distribution (9): $b_{n}$ is computed with Lambert function, $b_{n}^{\prime}$ is the standard constant (10), and $b_{n}^{\prime \prime}$ is the proposed constant (17).

We see in Section 4 that when we consider a generalized Weibull distribution with $\tau>1$, the use of $a_{n}^{\prime \prime}$ and $b_{n}^{\prime \prime}$ is practically indistinguishable from the use of $a_{n}$ and $b_{n}$.

\subsection{The simulation approach}

Random variables with distribution function given by (9) can be simulated by inversion method because the quantile function corresponding to $F$ is explicit in terms of the Lambert $W$ function,

$$
F^{-1}(u)=-W_{-1}((u-1) / e), u \in(0,1) .
$$

The random variables used to construct the histograms in Figure 3 have been simulated in this way.

\section{Generalized Weibull distribution}

In this section we deal with a sample of a generalized Weibull distribution as presented in Section 2. Let $a_{n}$ and $b_{n}$ be given by (7). In the following theorem we prove that when we use these constants, the order of convergence of the normalized maxima to the Gumbel distribution when $\tau>1$ is $1 / \log n$ (for $\tau=1$ is of order $1 / \log ^{2} n$ ). However, if we use another pair of admissible constants $\widetilde{a}_{n}$ and $\widetilde{b}_{n}$ (with some restrictions) then the order of convergence decreases to the lowest order between $b_{n}^{\tau}-\widetilde{b}_{n}^{\tau}$ and $1 / \log n$. As a consequence, 


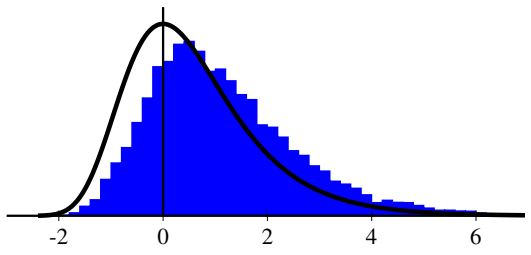

(a) With standard norming constants $a_{n}^{\prime}$ and $b_{n}^{\prime}$

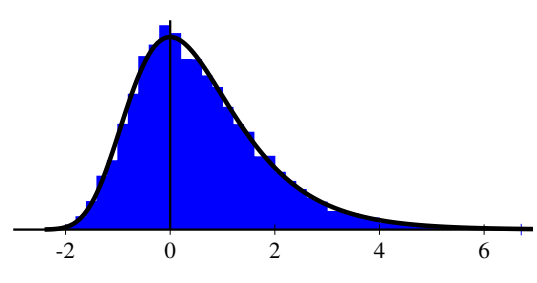

(b) With proposed norming constants $a_{n}^{\prime \prime}$ and $b_{n}^{\prime \prime}$

Fig. 3: Solid line: Gumbel density. Histograms of a simulation of $10^{4}$ normalized maxima with $n=100$, of a generalized Weibull distribution of parameters $C=\tau=\alpha=1$, with two different sets of norming constants

the nearer the constant $\widetilde{b}_{n}$ to $b_{n}$, the better the order of convergence. Given that $b_{n}$ can be expressed explicitly in terms of the Lambert $W$ function, it can be proposed, in terms of the asymptotics of that function, new constants that significantly improve the velocity of convergence.

Theorem 1 Let $X_{1}, \ldots, X_{n}$ be i.i.d. random variables with generalized Weibull distribution, with distribution function

$$
F(x)=1-K x^{\alpha} \exp \left\{-C x^{\tau}\right\}, x \geq x_{0},
$$

with $K, C, \alpha>0$ and $\tau \geq 1$.

1. Let $b_{n}=F^{-1}\left(1-n^{-1}\right)$ and $a_{n}=\left(C \tau b_{n}^{\tau-1}-\alpha / b_{n}\right)^{-1}$. Then, when $n \rightarrow \infty$,

$$
F^{n}\left(a_{n} x+b_{n}\right)-\Lambda(x)= \begin{cases}g_{1}(x) / \log n+O\left(\log \log n / \log ^{2} n\right), & \text { if } \tau>1, \\ g_{2}(x) / \log ^{2} n+O\left(\log \log n / \log ^{3} n\right), & \text { if } \tau=1,\end{cases}
$$

where $\Lambda(x)=\exp \left\{-e^{-x}\right\}$ is the distribution function of the Gumbel law, the functions $g_{1}$ and $g_{2}$ are bounded, and the big $O$ terms depend on $x$.

2. If $\widetilde{a}_{n}$ and $\widetilde{b}_{n}$ satisfy

$$
\widetilde{b}_{n}^{\tau}=b_{n}^{\tau}+T_{n} \quad \text { and } \quad \widetilde{a}_{n}=1 /\left(C \tau \widetilde{b}_{n}^{\tau-1}\right)+O\left(1 / \widetilde{b}_{n}^{2 \tau-1}\right),
$$

where $\lim _{n} \log n T_{n}^{2}=0$ and $\lim _{n} n T_{n}^{2}=\infty$, then, when $n \rightarrow \infty$,

$$
F^{n}\left(\widetilde{a}_{n} x+\widetilde{b}_{n}\right)-\Lambda(x)=h(x) T_{n}+O(1 / \log n) .
$$

where the function $h$ is bounded, and the big $O$ term depends on $x$.

\section{Remark 2}

1. We prove in Subsection 4.1 that the standard constants (8) as well as the new ones proposed in Subsection 4.3 satisfy the conditions (18).

2. When $\tau=1$ and $\widetilde{a}_{n}=\left(C-\alpha / \widetilde{b}_{n}\right)^{-1}$, it can be seen that

$$
F^{n}\left(\widetilde{a}_{n} x+\widetilde{b}_{n}\right)-\Lambda(x)=h(x) T_{n}+O\left(T_{n}^{2}\right)+O\left(1 / \log ^{2} n\right) .
$$


We need the following lemma:

Lemma 1 Let $B \neq 0$ and consider a sequence $\left\{c_{n}, n \geq 1\right\}$ such that $\lim _{n} c_{n}=$ 0 and $\lim _{n} n c_{n}^{2}=\infty$. Then, when $n \rightarrow \infty$,

$$
\left(1+B\left(1+c_{n}\right) / n\right)^{n}=e^{B}\left(1+B c_{n}+O\left(c_{n}^{2}\right)\right) .
$$

Proof of the lemma. From the fact that $\log (1+x)=x+O\left(x^{2}\right)$, when $x \rightarrow 0$, we get

$$
n \log \left(1+B\left(1+c_{n}\right) / n\right)=B+B c_{n}+O(1 / n) .
$$

Finally, using that $e^{x}=1+x+O\left(x^{2}\right)$, when $x \rightarrow 0$, and that $\lim _{n} n c_{n}^{2}=\infty$ the lemma follows.

Proof of Theorem 1.

1. We have

$$
\begin{aligned}
K\left(a_{n} x+\right. & \left.b_{n}\right)^{\alpha} \exp \left\{-C\left(a_{n} x+b_{n}\right)^{\tau}\right\} \\
& =K b_{n}^{\alpha} \exp \left\{-C b_{n}^{\tau}\right\}\left(\frac{a_{n}}{b_{n}} x+1\right)^{\alpha} \exp \left\{-C\left(a_{n} x+b_{n}\right)^{\tau}+C b_{n}^{\tau}\right\} \\
& \stackrel{(*)}{=} \frac{1}{n}\left(\frac{a_{n}}{b_{n}} x+1\right)^{\alpha} \exp \left\{-C\left(a_{n} x+b_{n}\right)^{\tau}+C b_{n}^{\tau}\right\} \\
& =\frac{e^{-x}}{n}\left(1+\alpha \frac{a_{n}}{b_{n}} x+O\left(\frac{a_{n}^{2}}{b_{n}^{2}}\right)\right) \\
& \times \exp \left\{-C b_{n}^{\tau}\left(1+\tau \frac{a_{n}}{b_{n}} x+\left(\begin{array}{l}
\tau \\
2
\end{array}\right) \frac{a_{n}^{2} x^{2}}{b_{n}^{2}}+O\left(\frac{a_{n}^{3}}{b_{n}^{3}}\right)-1\right)+x\right\} \\
& \stackrel{(* *)}{=} \frac{e^{-x}}{n}\left(1+R(x) \frac{1}{b_{n}^{\tau}}+O\left(\frac{1}{b_{n}^{2 \tau}}\right)\right),
\end{aligned}
$$

where equality $(*)$ follows from the definition of $b_{n}$, equality $(* *)$ is due to

$$
\frac{a_{n}}{b_{n}}=\frac{1}{C \tau b_{n}^{\tau}}+\frac{\alpha}{C^{2} \tau^{2} b_{n}^{2 \tau}}+O\left(\frac{1}{b_{n}^{3 \tau}}\right),
$$

and $R(x)=(1-\tau) x^{2} /(2 C \tau)$. We apply Lemma 1 with $B=-e^{-x}$ and $c_{n}=$ $R(x) / b_{n}^{\tau}+O\left(1 / b_{n}^{2 \tau}\right)$. Now we use that $b_{n}^{\tau}=C^{-1} \log n+O(\log \log n)$, which is proved in Subsection 4.1. Then $\lim _{n} n c_{n}^{2}=\infty$. Hence,

$$
\begin{aligned}
F^{n}\left(a_{n} x+b_{n}\right) & =\left(1-K\left(a_{n} x+b_{n}\right)^{\alpha} \exp \left\{-C\left(a_{n} x+b_{n}\right)^{\tau}\right\}\right)^{n} \\
& =\Lambda(x)\left(1-e^{-x} R(x) / b_{n}^{\tau}+O\left(1 / b_{n}^{2 \tau}\right)\right. \\
& =\Lambda(x)\left(1-e^{-x} C R(x) / \log n+O\left(\log \log n / \log ^{2} n\right)\right) .
\end{aligned}
$$

Finally, note that the function $R(x) \Lambda(x) e^{-x}$ is bounded.

If $\tau=1$, we have that $R(x)=0$, and it is needed to take one more term in the asymptotic expansions in order to make explicit the coefficient of $1 / b_{n}^{2}$. 
2. First note that from hypothesis (18) and the conditions on $T_{n}$ it is deduced that $\widetilde{b}_{n}^{\tau} \sim b_{n}^{\tau} \sim C^{-1} \log n$. Proceeding as before,

$$
\begin{aligned}
K\left(\widetilde{a}_{n} x\right. & \left.+\widetilde{b}_{n}\right)^{\alpha} \exp \left\{-C\left(\widetilde{a}_{n} x+\widetilde{b}_{n}\right)^{\tau}\right\} \\
& =\frac{e^{-x}}{n}\left(\frac{\widetilde{b}_{n}}{b_{n}}\right)^{\alpha}\left(1+\frac{\widetilde{a}_{n}}{\widetilde{b}_{n}} x\right)^{\alpha}\left(1-C\left(\widetilde{b}_{n}^{\tau}-b_{n}^{\tau}\right)+O\left(\frac{1}{\widetilde{b}_{n}^{\tau}}\right)\right) \\
& =\frac{e^{-x}}{n}\left(\frac{\widetilde{b}_{n}}{b_{n}}\right)^{\alpha}\left(1-C T_{n}+O\left(\frac{1}{\log n}\right)\right)=\frac{e^{-x}}{n}\left(1-C T_{n}+O\left(\frac{1}{\log n}\right)\right) .
\end{aligned}
$$

As in part 1 of this proof, applying Lemma 1 with $B=-e^{-x}$ and $c_{n}=$ $-C T_{n}+O(1 / \log n)$, we obtain the desired result.

4.1 Computation of the constants using Lambert $W$ function

The equation $b_{n}=F^{-1}\left(1-n^{-1}\right)$ is equivalent to

$$
K b_{n}^{\alpha} \exp \left\{-C b_{n}^{\tau}\right\}=1 / n .
$$

In terms of the Lambert $W$ function the solution is

$$
b_{n}=\left(-\frac{\alpha}{C \tau} W_{-1}\left(-\frac{C \tau}{\alpha(K n)^{\tau / \alpha}}\right)\right)^{1 / \tau} .
$$

From the asymptotic expansion of the Lambert function (11),

$$
b_{n}=\left(\frac{\alpha}{C \tau}\right)^{1 / \tau}\left(-M_{1}+M_{2}-\frac{M_{2}}{M_{1}}+\cdots\right)^{1 / \tau},
$$

where

$$
M_{1}=\log \left(\frac{C \tau}{\alpha(K n)^{\tau / \alpha}}\right) \quad \text { and } \quad M_{2}=\log \left(-M_{1}\right)
$$

In particular, we have $b_{n}^{\tau}=C^{-1} \log n+O(\log \log n)$.

\subsection{Computation of the constants using Comtet expansion}

The solution of equation (19) can be expressed in an alternative way: Fix $\gamma \neq 0$ and denote by $U_{\gamma}(x)$ the (unique) solution of the equation $t^{\gamma} e^{t}=x$ such that $\lim _{x \rightarrow \infty} U_{\gamma}(x)=\infty$. Equation (19) is equivalent to $b_{n}^{-\alpha} e^{C b_{n}^{\tau}} / K=n$. Hence,

$$
b_{n}=\left(\frac{1}{C} U_{-\alpha / \tau}\left(\frac{K n}{C^{\alpha / \tau}}\right)\right)^{1 / \tau} .
$$


Comtet [1] extended De Bruijn expansion (11) to $U_{\gamma}$ obtaining

$$
U_{\gamma}(x)=L_{1}(x)-\gamma L_{2}(x)+\sum_{n=1}^{\infty}(-\gamma)^{n+1} \frac{P_{n}\left(L_{2}(x)\right)}{L_{1}^{n}(x)}, x \rightarrow \infty,
$$

where $L_{1}(x), L_{2}(x)$ are given in (12), and $P_{n}(x)$ are polynomials related to the signed Stirling numbers of the first type; the first three polynomials are

$$
P_{1}(x)=x, P_{2}(x)=x^{2} / 2-x \quad \text { and } \quad P_{3}(x)=x^{3} / 3-3 x^{2} / 2+x .
$$

Note that the asymptotic expansion of the Lambert function (11) can be written in terms of these polynomials. Applying that expansion to (22) we get a new asymptotic expansion for $b_{n}$; specifically,

$$
b_{n}=\frac{1}{C^{1 / \tau}}\left(N_{1}+\frac{\alpha}{\tau} N_{2}+\frac{\alpha^{2}}{\tau^{2}} \frac{N_{2}}{N_{1}}+\cdots\right)^{1 / \tau},
$$

where

$$
N_{1}=\log \left(K n / C^{\alpha / \tau}\right) \text { and } N_{2}=\log \left(N_{1}\right) .
$$

Although the whole sum of both series (20) and (24) is the same, the finite expansions obtained by truncation are slightly different; the difference is due to some constants appearing early in the former are delayed in the latter. It turns out that when $\alpha>\tau$, it is better the truncation from (20), when $\alpha<\tau$ it is better the approximation given by (24), and when $\alpha=\tau$ both expansions coincide. See Online Appendix 2 for a proof.

\subsection{Proposed new constants}

The standard constant $b_{n}^{\prime}$ given in (8) corresponds to take the terms $N_{1}+$ $(\alpha / \tau) N_{2}$ in $(24)$, and it is deduced, with the notations of Theorem 1 , that $T_{n}=\left(b_{n}^{\prime}\right)^{\tau}-b_{n}^{\tau} \sim A_{1}(\log \log n)^{2} / \log n$ for some constant $A_{1}$. Hence, the conditions on $T_{n}$ and $a_{n}^{\prime}$ given in (18) of Theorem 1 are satisfied. This implies that the velocity of convergence of the maxima to the Gumbel law with the standard constants is of order $(\log \log n)^{2} / \log n$. However, we propose to take one more term of that expansion. Specifically,

- If $\alpha>\tau$,

$$
b_{n}^{\prime \prime}=\left(\frac{\alpha}{C \tau}\right)^{1 / \tau}\left(-M_{1}+M_{2}-\frac{M_{2}}{M_{1}}\right)^{1 / \tau},
$$

and

$$
a_{n}^{\prime \prime}=\frac{1}{C \tau\left(b_{n}^{\prime \prime}\right)^{\tau-1}-\alpha / b_{n}^{\prime \prime}},
$$

where $M_{1}$ and $M_{2}$ are defined by (21).

- If $\alpha \leq \tau$,

$$
b_{n}^{\prime \prime}=\frac{1}{C^{1 / \tau}}\left(N_{1}+\frac{\alpha}{\tau} N_{2}+\frac{\alpha^{2}}{\tau^{2}} \frac{N_{2}}{N_{1}}\right)^{1 / \tau},
$$


where $N_{1}$ and $N_{2}$ are defined in (25), and $a_{n}^{\prime \prime}$ the same as in (27).

It turns out that now $T_{n}=\left(b_{n}^{\prime \prime}\right)^{\tau}-b_{n}^{\tau} \sim A_{2}(\log \log n / \log n)^{2}$, so by Theorem 1 , if $\tau>1$, with these constants the order of convergence to the Gumbel law is $1 / \log n$, thus it is attained the same rate as with $a_{n}$ and $b_{n}$. If $\tau=1$, the order of convergence with $a_{n}$ and $b_{n}$ is is $1 / \log ^{2} n$, and by Remark 2 of Theorem 1 the order of convergence with $a_{n}^{\prime \prime}$ and $b_{n}^{\prime \prime}$ is $(\log \log n / \log n)^{2}$.

\subsection{A comment on the parameter space}

In this paper we only consider $\alpha>0$ and $\tau \geq 1$ for the generalized Weibull distribution given in (6); the motivation for the restriction $\alpha>0$ is that for $\alpha<0$, the expression of $b_{n}$ should be given in terms of the principal branch of Lambert $W$ function. On the other hand, for $0<\tau<1, \lim _{n} a_{n}=\infty$, and hence Theorem 1 should be adapted. The extension of the results to these cases would enlarge the paper and make the notations more complex.

\section{Maxima of Gamma distributions}

In this section we study the case when the sample comes from a Gamma distribution $G(\nu, \theta)$ with density function $f(x)=x^{\nu-1} \exp \{-x / \theta\} /\left(\theta^{\nu} \Gamma(\nu)\right), x>0$, with $\nu>1$ and $\theta>0$. Its distribution function $F$ can be written in terms of the incomplete Gamma function as

$$
F(x)=1-\Gamma(\nu, x / \theta) / \Gamma(\nu), x \geq 0,
$$

where $\Gamma(a)=\int_{0}^{\infty} t^{a-1} e^{-t} d t, a>0$, is the Gamma function, and $\Gamma(a, y)=$ $\int_{y}^{\infty} t^{a-1} e^{-t} d t, a, y>0$, is the upper incomplete Gamma function.

From formula $\lim _{y \rightarrow \infty} \Gamma(a, y) /\left(y^{a-1} e^{-y}\right)=1$, see Olver et al. [7, form. $8.11 .2]$, it follows

$$
\lim _{x \rightarrow \infty} \frac{1-F(x)}{K x^{\alpha} \exp \left\{-C x^{\tau}\right\}}=1,
$$

with $K=\left(\theta^{\nu-1} \Gamma(\nu)\right)^{-1}, \alpha=\nu-1, C=1 / \theta$ and $\tau=1$. So a Gamma law is Weibull-like. In agreement with our comments in Section 2, the standard norming constants (see Embrechts et al. [4, p. 155]) are

$$
b_{n}^{\prime}=\theta(\log n+(\nu-1) \log (\log n)-\log \Gamma(\nu)) \quad \text { and } \quad a_{n}^{\prime}=\theta .
$$

5.1 Main result: New norming constants for the maxima of Gamma and $\chi^{2}(m)$ distributions

Numerical computations show that the constants (31) produce very inaccurate results (see Subsection 5.3). Extending to this case the arguments used with 
the generalized Weibull distributions, for a Gamma law $G(\nu, \theta)$ we propose the following constants:

For $\nu \in(1,2]$ :

$$
\begin{aligned}
b_{n}^{\prime \prime}=\theta & (\log (n / \Gamma(\nu))+(\nu-1) \log \log (n / \Gamma(\nu)) \\
& \left.+\left((\nu-1)^{2} \log \log (n / \Gamma(\nu))+\nu-1\right) / \log (n / \Gamma(\nu))\right),
\end{aligned}
$$

and

$$
a_{n}^{\prime \prime}=b_{n}^{\prime \prime} \theta\left(b_{n}^{\prime \prime}+\theta(\nu-1)\right) /\left(b_{n}^{\prime \prime 2}-\theta^{2}(\nu-1)(\nu-2)\right) .
$$

For $\nu \geq 2$ :

$$
\begin{aligned}
b_{n}^{\prime \prime}=\theta & \left(\log (n / \Gamma(\nu))+(\nu-1) \log B_{n}\right. \\
& \left.+\left((\nu-1)^{2} \log B_{n}-(\nu-1)^{2} \log (\nu-1)+\nu-1\right) / B_{n}\right),
\end{aligned}
$$

where

$$
B_{n}=\log (n / \Gamma(\nu))+(\nu-1) \log (\nu-1),
$$

and $a_{n}^{\prime \prime}$ the same as (32).

Since a $\chi^{2}(m)$ law is a Gamma law $G(\nu, \theta)$ with $\nu=m / 2$ and $\theta=2$, the constants for the maxima of $n$ random variables with $\chi^{2}(m)$ law are easily deduced.

\subsection{Deduction of the new norming constants}

To the best of our knowledge, for a Gamma law (except when $\nu=2$, see Remark 3 below) the quantile function $F^{-1}$ cannot be written in closed form in terms of the Lambert function. However, given the asymptotic result (30), the generalized Weibull distribution function

$$
F_{1}(x)=1-x^{\nu-1} \exp \{-x / \theta\} /\left(\theta^{\nu-1} \Gamma(\nu)\right), x \geq x_{0},
$$

is tail equivalent to the Gamma law $G(\nu, \theta)$. Hence, by Property 1, the constants deduced in Section 4 could be used to try to improve the velocity of convergence. However, in this case, the simple addition of more terms using Comtet or Lambert expansion does not give enough improvement and therefore we consider a right tail equivalent distribution function more accurate than $F_{1}$. We will use that the incomplete Gamma function $\Gamma(a, y)(a>0)$ has the following asymptotic expansion for $y \rightarrow \infty$ (see Olver et al. [7, form. 8.11.2]):

$$
\Gamma(a, y) \sim y^{a-1} e^{-y}\left(1+(a-1) / y+(a-1)(a-2) / y^{2}+\cdots\right) .
$$


Observe that when $a$ is an integer, the series in the right hand side terminates, and the expression is not only asymptotic but exact for all $y>0$; this is what happens with the distribution function of a $\chi^{2}(m)$ random variable with $m$ even. So, we will consider a distribution function of the form

$$
F_{2}(x)=1-x^{\nu-1} \exp \{-x / \theta\}(1+\theta(\nu-1) / x) /\left(\theta^{\nu-1} \Gamma(\nu)\right), x \geq x_{0} .
$$

The auxiliar function (5) corresponding to $F_{2}$ is

$$
A_{2}(x)=x \theta(x+\theta(\nu-1)) /\left(x^{2}-\theta^{2}(\nu-1)(\nu-2)\right) .
$$

In contrast with $F_{1}$ the quantile function $F_{2}^{-1}$, unless $\nu=2$, has no explicit expression in terms of the Lambert function; however, a fine asymptotic expansion can be obtained, and this gives us a way to propose the new constants. In next subsections we see extensions of Lambert and Comtet asymptotic expansions to this new context.

Remark 3 The distribution function of a Gamma $G(2, \theta)$ law is $F(x)=1-$ $(1+x / \theta) e^{-x / \theta}, x \geq 0$; note that it is of type $F_{2}$ given in (34) above. Jiménez and Jodrá [6] prove that its quantile function can be written in terms of the Lambert $W$ function. Thanks to this property, the study of the norming constants can be reduced to the analysis in Section 3. Specifically, set $F_{0}(x)=1-e x e^{-x}$ the distribution function introduced there, and let $b_{n}^{\circ}$ and $a_{n}^{\circ}$ be the corresponding constants given in (14) and (16) respectively. Note that $F(x)=F_{0}(1+x / \theta)$. Consider the constants $b_{n}$ and $a_{n}$ of $F$ given in (3), (4), and (5). From Jiménez and Jodrá [6, Prop. 1],

$$
b_{n}=F^{-1}(1-1 / n)=-\theta\left(W_{-1}(-1 /(n e))+1\right)=\theta\left(b_{n}^{\circ}-1\right),
$$

where the last equality follows from (14). Also, $a_{n}=\theta+\theta^{2} / b_{n}=\theta a_{n}^{\circ}$. Then

$$
F\left(a_{n} x+b_{n}\right)=F_{0}\left(a_{n}^{\circ} x+b_{n}^{\circ}\right) .
$$

Hence, the results of Theorem 1, Part $1(\tau=1)$, can be applied to a $G(2, \theta)$ law. Similarly, with the notations of that theorem, taking $\widetilde{b}_{n}=b_{n}+T_{n}$ and $\widetilde{a}_{n}=A_{2}\left(\widetilde{b}_{n}\right)=\theta+\theta^{2} / \widetilde{b}_{n}$, where $A_{2}$ is given in (35), Remark 2.2 also holds in this case.

\subsubsection{Extension of Comtet expansion}

Robin [9] and Salvi [10] extended Comtet [1] results to the deduction of an asymptotic expansion of the solution of the equation

$$
t^{\gamma} e^{t} D(1 / t)=x
$$

such that $t \rightarrow \infty$ when $x \rightarrow \infty$, where $\gamma \neq 0$ and

$$
D(t)=\sum_{n=0}^{\infty} d_{n} t^{n}, \text { with } d_{0} \neq 0,
$$


is a power series convergent in a neighborhood of the origin. Denote by $U_{\gamma, D}(x)$ that solution. Robin [9] and Salvi [10] prove that for every $N$, for $x \rightarrow \infty$,

$$
U_{\gamma, D}(x)=L_{1}(x)+\sum_{n=0}^{N} \frac{Q_{n}\left(L_{2}(x)\right)}{L_{1}^{n}(x)}+o\left(\frac{1}{L_{1}^{N}(x)}\right),
$$

where $L_{1}$ and $L_{2}$ are the same as in (12), and $Q_{n}(x)$ are polynomials depending on $\gamma$ and on the series $D, Q_{n}=Q_{n}\left(\gamma, d_{0}, \ldots, d_{n}\right)$, with degree $n$ for $n \geq 1$, and $Q_{0}$ of degree 1 . The first two polynomials (fortunately, the only ones that we need), see Online Appendix 2, are

$$
Q_{0}(x)=-\gamma x-\log d_{0} \quad \text { and } \quad Q_{1}(x)=\gamma^{2} x+\gamma \log d_{0}-d_{1} / d_{0} .
$$

When $D(x)=1$, then $Q_{0}(x)=-\gamma x$, and for $n \geq 1, Q_{n}(x)=(-\gamma)^{n+1} P_{n}(x)$, where $P_{n}$ are the polynomials in Subsection 4.2.

\subsubsection{Extension of Lambert expansion}

Consider the equation (36) for $\gamma=1$. The inverse of the function

$$
f(t)=t e^{t} D(1 / t)
$$

( $t$ out of a neighborhood of the origin) has a secondary branch, denoted by $W_{-1, D}(x)$, that goes to $-\infty$ when $x \rightarrow 0^{-}$. The asymptotic expansion of this branch (see Online Appendix 2) is

$$
W_{-1, D}(x)=L_{1}(-x)+\sum_{n=0}^{\infty}(-1)^{n+1} R_{n}\left(L_{2}(-x)\right) / L_{1}^{n}(-x) .
$$

The first two polynomials are

$$
R_{0}(x)=x+\log d_{0} \quad \text { and } \quad R_{1}(x)=x+\log d_{0}-d_{1} / d_{0} .
$$

The relationship between the polynomials $Q_{n}$ of Subsection 5.2.1 and $R_{n}$ is studied in the Online Appendix 2.

\subsubsection{Last step}

As we commented, we apply the principle of simplification by tail equivalence given in Property 1, and from (7) for the distribution function $F_{2}$ given in $(34), b_{n}$ verifies

$$
\left(b_{n} / \theta\right)^{\nu-1} \exp \left\{-b_{n} / \theta\right\}\left(1+\theta(\nu-1) / b_{n}\right)=\Gamma(\nu) / n .
$$

As a consequence of the previous two subsections, we have two ways to express the solution of this equation. First, writing $y=b_{n} / \theta$, we need to solve

$$
y^{\nu-1} e^{-y}(1+(\nu-1) / y)=\Gamma(\nu) / n .
$$


Hence, with the notation of Subsection 5.2.1,

$$
b_{n}=\theta U_{1-\nu, D}(n / \Gamma(\nu)),
$$

where $D(t)=(1+(\nu-1) t)^{-1}=1-(\nu-1) t+O\left(t^{2}\right)$.

Second, in a similar way, we can transform equation (41),

$$
(-y /(\nu-1)) e^{-y /(\nu-1)}(1-1 /(-y /(\nu-1)))^{1 /(\nu-1)}=(\Gamma(\nu) / n)^{1 /(\nu-1)} /(1-\nu) .
$$

Thus, with the notation of Subsection 5.2.2,

$$
b_{n}=-(\nu-1) \theta W_{-1, E}\left((\Gamma(\nu) / n)^{1 /(\nu-1)} /(1-\nu)\right),
$$

where $E(t)=(1-t)^{1 /(\nu-1)}=1-t /(\nu-1)+O\left(t^{2}\right)$.

We prove in Online Appendix 2 that the finite expansions deduced from (42) and (43) when $\nu=2$ are equal, when $\nu>2$ then (43) gives a more accurate value, and when $1<\nu<2$ it is better to use the expansion deduced from (42). As in Section 3, we added just one more term of the asymptotic expansion of $b_{n}$; numerical results show that the approximation to the Gumbel law is much better than with the standard constants, see next subsection. Hence, we consider only the polynomials $Q_{0}$ and $Q_{1}$, that depend on the terms $d_{0}$ and $d_{1}$ of the series $D(t)$ or $E(t)$. The formulas given in Subsection 5.1 for $1<\nu \leq 2$ are deduced from (42), (37) and (38); for $\nu \geq 2$ the formulas follow from (43), (39) and (40).

\subsection{Numerical comparison}

In this subsection we analyze numerically the case of a $\chi^{2}(10)$ distribution; Similar results are obtained for the case $\nu \in(1,2)$, for example, for a $\chi^{2}(3)$ distribution. however, in this last case the discrepancy between the approximation using the standard constants and the Gumbel distribution is not as serious as in the case $\nu \geq 2$.

In Table 2 the numeric value of $b_{n}$ given in (3) corresponding to a $\chi^{2}(10)$ distribution is computed using the quantile function of a $\chi^{2}$ distribution implemented in $\mathbf{R}$ program. The constant $b_{n}^{\prime}$ is the standard value (31) and $b_{n}^{\prime \prime}$ is the value (33).

\begin{tabular}{ccccccc}
\hline $\boldsymbol{n}$ & 10 & $10^{2}$ & $10^{3}$ & $10^{4}$ & $10^{5}$ & $10^{6}$ \\
$b_{n}$ & 15.9872 & 23.2093 & 29.5883 & 35.5640 & 41.2962 & 46.8630 \\
$b_{n}^{\prime}$ & 4.9213 & 15.0717 & 22.9606 & 29.8272 & 36.2175 & 42.2812 \\
$b_{n}^{\prime \prime}$ & 13.3518 & 22.0874 & 29.0421 & 35.2855 & 41.1581 & 46.8045 \\
\hline
\end{tabular}

Table 2: Comparison of the constants $b_{n}$ for the $\chi^{2}(10)$ distribution: $b_{n}$ is the numeric value, $b_{n}^{\prime}$ is the standard value given in $(31), b_{n}^{\prime \prime}$ is the new proposed constant given in (33). 
For the constant $a_{n}$ there are also important differences, see Table 3 . The value $a_{n}$ given in (4)-(5) is computed using the distribution function and the density function of a $\chi^{2}(10)$ law implemented in $\mathbf{R}$ program, $a_{n}^{\prime}$ is the standard value (31) and $a_{n}^{\prime \prime}$ is the value given in (32)

\begin{tabular}{rcccccc}
\hline $\boldsymbol{n}$ & 10 & $10^{2}$ & $10^{3}$ & $10^{4}$ & $10^{5}$ & $10^{6}$ \\
$a_{n}$ & 3.4821 & 2.9010 & 2.6662 & 2.5348 & 2.4495 & 2.3890 \\
$a_{n}^{\prime}$ & 2 & 2 & 2 & 2 & 2 & 2 \\
$a_{n}^{\prime \prime}$ & 4.3768 & 3.0217 & 2.7049 & 2.5518 & 2.4584 & 2.3943 \\
\hline
\end{tabular}

Table 3: Comparison of the constants $a_{n}$ for the $\chi^{2}(10)$ distribution: $a_{n}$ is the numeric value, $a_{n}^{\prime}$ is the standard value (31), $a_{n}^{\prime \prime}$ is the value given in (32).

In Figure 4 there is a plot of the density functions of the random variables

$$
Y_{n}=\left(M_{n}-b_{n}\right) / a_{n}, \quad Y_{n}^{\prime}=\left(M_{n}-b_{n}^{\prime}\right) / a_{n}^{\prime}, \text { and } \quad Y_{n}^{\prime \prime}=\left(M_{n}-b_{n}^{\prime \prime}\right) / a_{n}^{\prime \prime}
$$

from a sample of size $n=100$ of $\chi^{2}(10)$ random variables, where $b_{n}$ and $a_{n}$ are the numeric solutions of equations $(7), b_{n}^{\prime}$ and $a_{n}^{\prime}$ are the standard constants (31), $b_{n}^{\prime \prime}$ and $a_{n}^{\prime \prime}$ are the constants (33) and (32), respectively.

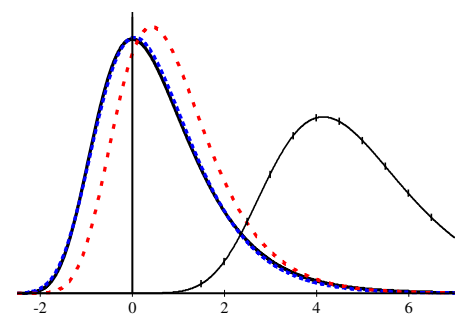

Fig. 4: Maximum of $100 \chi^{2}(10)$ random variables. Solid line: Gumbel density. Dashed Blue line: Density of $Y_{n}$. Loosely dashed red line: Density of $Y_{n}^{\prime \prime}$. Thin line with vertical marks: Density of $Y_{n}^{\prime}$.

\section{An example with real data}

In the Online Appendix 1 it is explained the problem that was in the origin of this work and some real data are given to illustrate the use of Extreme Value Theory in the context of signal processing. Those data were gathered with a GPS front-end at 09:22AM (CET) March 26, 2008, at Noordwijk (The Netherlands), and they correspond to the acquisition of the GPS satellite with PRN 11 using a software receiver implemented in Matlab. As exposed in that Online Appendix 1, we consider a sample of $n=10^{5}$ random variables. The 
core of the problem is to test whether the sample contains some signal or there is only noise; in this last case, all random variables have a $\chi^{2}(10)$ law. The null hypothesis $\mathcal{H}_{0}$ is that there is no signal. To perform the test, our best option (see Figure 4 above, or Figure 5 in the Online Appendix 1) is to use the normalized maximum $\left(M_{n}-b_{n}\right) / a_{n}$ as a test statistic, where $a_{n}$ and $b_{n}$ are the numeric solutions of equations (3), (4) and (5), whose values for this specific sample size appear in Tables 2 and 3 . Under $\mathcal{H}_{0}$, we can assume that this statistic has a Gumbel distribution. Fixed a significance level, say $\alpha=0.01$, with the help of the Gumbel distribution function $\Lambda$ it is found that the critical region is $(4.6002, \infty)$. However, if we use the standard constants $a_{n}^{\prime}$ and $b_{n}^{\prime}$ given in (31), the significance level increases dramatically,

$$
P\left(\left(M_{n}-b_{n}^{\prime}\right) / a_{n}^{\prime}>4.6002 \mid \mathcal{H}_{0}\right) \approx 1-\Lambda(1.6826) \approx 0.17 .
$$

If we use the proposed constants $a_{n}^{\prime \prime}$ and $b_{n}^{\prime \prime}$ (see again Tables 2 and 3), then the significance level is 0.0104 , which is practically the nominal value.

\section{Conclusions}

From an engineering point of view, the maxima of Gamma random variables plays a major role in many applications dealing with signal detection problems. This is the case of radar, sonar, or wireless systems based on spread spectrum technology (e.g. 3G mobile communications or GNSS). In these applications, detecting the presence of the received signal involves finding the maximum $M_{n}$ over a population of Gamma distributed random variables $X_{1}, \ldots, X_{n}$, which are spread over a time-frequency plane. From a statistical point of view, this problem falls into the field of Extreme Value Theory, and it is known that Gamma laws are in the domain of attraction for maxima of the Gumbel or double-exponential law. This law provides a closed-form expression for assessing type I errors, or probability of false alarm, when testing the null hypothesis (i.e. that there is no signal in the sample). Therefore, the Gumbel law becomes a key performance tool for the theoretical assessment of a myriad of engineering systems.

Nevertheless, the use of the Gumbel law requires the proper use of some norming constants $a_{n}$ and $b_{n}$; since in general these constants do not have a closed form, in practice it is necessary to have some approximate explicit expressions of them. While some expressions are already available, which we call the standard constants, they suffer from two main drawbacks: first, they implicitly assume that the population size goes to $\infty$, when in practice applications deal with a finite $n$; second, there is no guarantee that these standard constants may provide a tighter match to the Gumbell law than some other approximate constants computed by some other means. We also checked numerically that these standard constants produce quite inaccurate results for moderate or even large sample size. This implies that systems designed with those constants tend to exhibit, in practice, a performance worse than the one initially expected. 
Based on the above observations, and for a family of distribution called generalized Weibull distributions, we have shown that standard norming constants can be significantly improved by adding one more term from the asymptotic expansion of the theoretical centering constant $b_{n}$. This asymptotic expansion is deduced by means of the Lambert $W$ function and its generalizations. These new constants have been shown to be much more accurate than the existing ones, and they preserve this property even for large population sizes of the order of $n=10^{6}$. These results are extended later to the Gamma laws, which are right tail equivalent to a generalized Weibull distribution.

\section{Acknowledgments}

The first author was partially supported by grants MINECO reference MTM 2013-40998-P and Generalitat de Catalunya reference 2014-SGR568. The second author by the European Space Agency (ESA) through the DINGPOS contract AO/1-5328/06/NL/GLC, and by the Spanish Government and Generalitat de Catalunya through grants TEC2011-28219 and 2014-SGR1586, respectively. The third author by grants MINECO reference MTM2012-33937 and Generalitat de Catalunya reference 2014-SGR422.

The authors thank two anonymous referees for their careful reading of the manuscript and their suggestions that helped to improve the paper.

\section{References}

1. Comtet, L (1970) Inversion de $y^{\alpha} e^{y}$ et $y \log ^{\alpha} y$ au moyen des nombres de Stirling. C. R. Acad. Sc. Paris, Serie A, 270:1085-1088

2. Corless RM, Gonnet GH, Hare DEG, Jeffrey DJ, Knuth DE (1996) On the Lambert $W$ function. Adv. Comput. Math. 5:329-359

3. De Bruijn N G (1981) Asymptotic Methods in Analysis. Dover, New York

4. Embrechts P, Klüppelberg C, Mikosch T (1997) Modeling Extremal Events for Insurance and Finance. Springer, Berlin

5. Hall P (1979) On the rate of convergence of normal extremes. J. Appl. Probab. 16:433439

6. Jiménez F, Jodrá P (2009) On the computer generation of the Erlang and negative binomial distributions with shape parameter equal to two. Math. Comput. Simulation 79:1636-1640

7. Olver FWJ, Lozier DW, Boisvert RF, Clark CW (2010) The NIST Handbook of Mathematical Functions. Cambridge University Press, Cambridge

8. Resnick SI (1987) Extreme Values, Regular Variation, and Point Processes. Springer, Berlin

9. Robin G (1988) Permanence de relations de récurrence dans certains développements asymptotiques. Publications de l'Institut Mathématique, Nouv. sér., 43:17-25

10. Salvy B (1994) Fast computation of some asymptotic functional inverses. J. Symbolic Comput. 17:227-236

11. Seco-Granados G, López-Salcedo JA, Jiménez-Baños D, and López-Risueño G (2012) Challenges in indoor global navigation satellite systems. IEEE Signal Processing Magazine 29:108-131

12. Turunen S. (2007) Network Assistance. What will new GNSS Signals Bring to it? Inside GNSS, www.insidegnss.com, Spring 2007, 35-41 


\title{
Online Appendix 1 for TEST article \\ Maxima of Gamma random variables and other Weibull-like distributions and the Lambert $W$ function
}

\author{
A. Gasull, J. A LóPeZ-S Alcedo, F. Utzet
}

\section{Application to the acquisition of GPS satellite signals}

The motivation of this appendix is to illustrate the advantages of the proposed norming constants by using data from a real application. As already introduced in Section 1 of the paper, the present study originated from a mathematical problem in the context of satellite navigation. In that context, the goal is to determine the user's position by measuring the signal propagation delay between each visible satellite and the user receiver. In order to accurately measure this time delay, the receiver must tune its internal radio components to the actual signal parameters of each of the visible satellites. This means adjusting its internal oscillator in both time (i.e. to compensate for the signal propagation delay) and frequency (i.e. to compensate for the Doppler effect and additional clock mismatches). The adjustment is typically carried out following a two-steps approach whereby a coarse adjustment is applied first, and then some refinements are applied next. These two steps are referred to as acquisition and tracking, respectively. The outputs of the tracking stage, for all the satellites in view, are used later on to find the so-called navigation solution, which brings an estimate of the current user's position, velocity and time. The whole process is schematically represented in Figure 1, where the block labeled "frontend" is in charge of the signal conditioning (i.e. filtering, amplification and analog-to-digital conversion of the received signal), and the rest of blocks are the acquisition, tracking and navigation modules mentioned before.

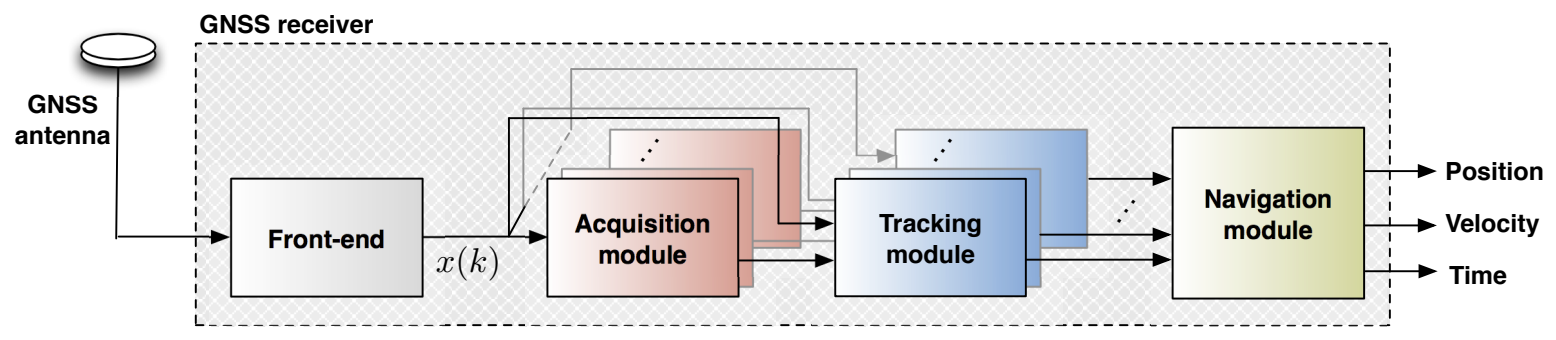

Figure 1. Schematic representation of the tasks that are involved in a generic Global Navigation Satellite System (GNSS) receiver.

We will focus herein on the acquisition module, whose inner constituent elements are schematically represented in Figure 2. Succinctly, the radio-frequency signal impinging onto the receiver antenna is conditioned first at the front-end module. The result is a stream of complex-valued discrete-time samples at the front-end output, denoted by $x(k)$, with $k$ the discrete-time indexation. In order to detect the visible satellites, the receiver must perform the correlation between a batch of $K$ samples of the received signal and a locally generated signal replica for the satellite of interest, denoted by $c(k)$. Assuming that we are processing the $i$-th batch of received samples, the resulting correlation can be expressed as:

$$
Z_{i}(\hat{f}, \hat{\tau}):=\sum_{k=0}^{K-1} x(i K+k) c(k-\hat{\tau}) e^{j 2 \pi \hat{f} k}
$$

The correlation in (1) is evaluated at a given tentative time-delay $\hat{\tau}$ and frequency shift $\hat{f}$ of the local signal replica $c(k)$. This is done in order to tentatively tune the receiver to the time-delay and frequency shift of the 
actual received signal. In order to improve the detection sensitivity, it is common to extend the correlation time by noncoherently accumulating $N_{I}$ results from (1). This leads to the following metric,

$$
X\left(\hat{f}_{p}, \hat{\tau}_{q}\right):=\sum_{i=1}^{N_{I}}\left|Z_{i}(\hat{f}, \hat{\tau})\right|^{2}
$$

which can be understood as an estimate of the received energy at the tentative pair of values $\{\hat{f}, \hat{\tau}\}$. The process in (2) is repeated using a different pair of tentative time-delay and frequency values, until the correct combination is found. Note that by "correct" we mean the pair of values actually used by the received signal, and denoted herein by $\left\{f_{*}, \tau_{*}\right\}$. This leads the receiver to implement a bi-dimensional search in the time/frequency plane, where a set of discrete values $\hat{f}_{p}$, for $p=1, \ldots, L_{f}$, and $\hat{\tau}_{q}$, for $q=1, \ldots, L_{\tau}$ are evaluated. We will refer to this bi-dimensional set of discrete values as the acquisition search space, denoted by $\mathcal{S}$ :

$$
\mathcal{S}=\left\{\left\{\hat{f}_{p}\right\}_{p=1}^{L_{f}},\left\{\hat{\tau}_{q}\right\}_{p=1}^{L_{\tau}}\right\}
$$

The result of the noncoherent correlation in (2), for each of the possible pair of values $\left\{\hat{f}_{p}, \hat{\tau}_{q}\right\}$, is stored into the $(p, q)$ entry of an $\left(L_{f} \times L_{\tau}\right)$ matrix $\mathbf{X}$, also known as the acquisition matrix. That is, $[\mathbf{X}]_{p, q}=X\left(\hat{f}_{p}, \hat{\tau}_{q}\right)$. Then, the final decision on whether the satellite under analysis is present or not boils down to deciding whether the maximum of the entries in $\mathbf{X}$ exceeds a given threshold or not. That is, we decide the satellite is present whenever

$$
\max _{p, q}[\mathbf{X}]_{p, q}>\gamma
$$

for some threshold $\gamma$, and that the satellite is not present, otherwise. These two situations are often referred to as the signal present or $\mathcal{H}_{1}$ hypothesis, and the signal absent or $\mathcal{H}_{0}$ hypothesis. Furthermore, note that the problem above can equivalently be posed as

$$
M_{n}=\max \left\{X_{1}, \ldots, X_{n}\right\}>\gamma,
$$

which was the starting point of the paper. To do so, $n:=L_{f} L_{\tau}$ becomes the total number of elements in $\mathbf{X}$, and $X_{l}$ for $l=1, \ldots, n$ is a simple way to denote the elements contained within that matrix.

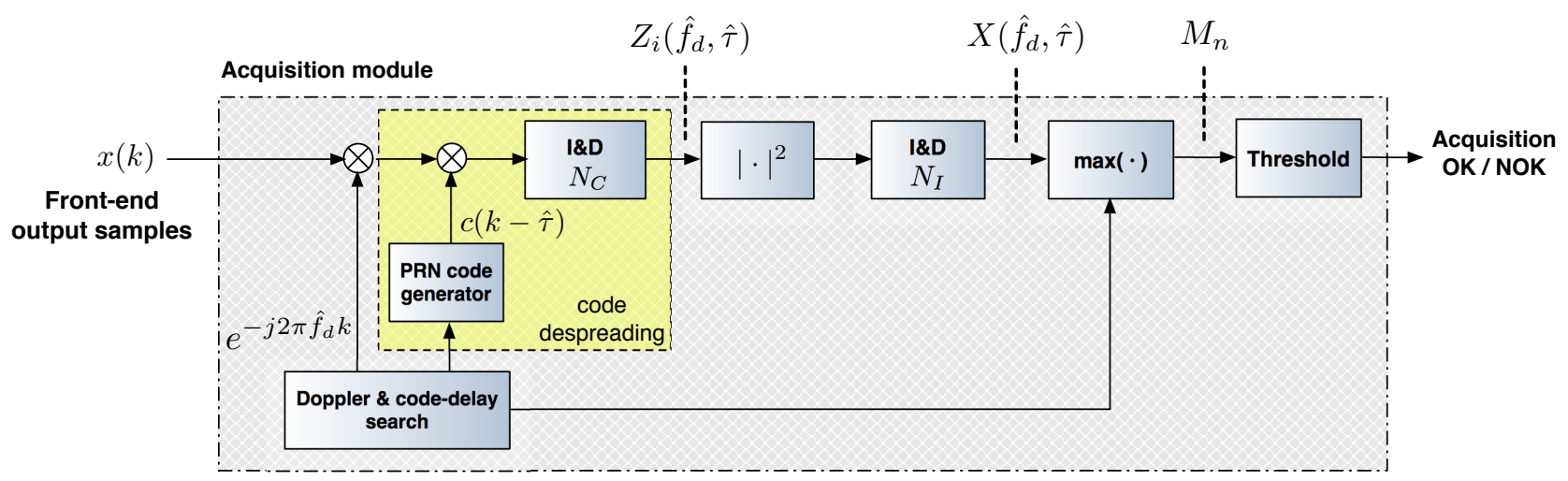

Figure 2. Schematic representation of the tasks that are involved in the acquisition stage of a GNSS receiver.

So far, we have illustrated the parallelism between the acquisition of GNSS satellite signals and the maximum of a set of $n$ random variables, as stated in (5). The next step is to show that under $\mathcal{H}_{0}$, i.e. when the satellite is absent, these $n$ random variables follow a Gamma distribution, and in particular, a $\chi^{2}(m)$ law. This will establish the link between the problem at hand and the results provided in Section 5 of the paper. It is 
interesting to note that we focus herein on the $\mathcal{H}_{0}$ hypothesis because it is the one that determines the probability of type I errors of the satellite acquisition process, or equivalently, the so-called probability of false alarm $\left(P_{F A}\right)$. This probability is a key design metric of any GNSS receiver, and therefore it is essential to have a very accurate statistical characterization.

To better illustrate the properties of the entries populating the acquisition matrix $\mathbf{X}$, i.e. the random variables $X_{1}, \ldots, X_{n}$ in (5), let us show some examples based on real measurements. These real data were gathered with a GPS front-end at 09:22AM (CET) March 26, 2008, at Noordwijk (The Netherlands), and they correspond to the acquisition of the GPS satellite with PRN 11 using a software receiver implemented in Matlab. Two different situations are shown in the two plots of Figure 3. The results on the left hand side correspond to the $\mathcal{H}_{1}$ hypothesis, where the true pair of frequency and time-delay values of the received signal $\left\{f_{*}, \tau_{*}\right\}$ are contained within the search space $\mathcal{S}$. In that case, the entry of $\mathbf{X}$ with the closest pair of tentative values $\left\{\hat{f}_{p}, \hat{\tau}_{q}\right\}$ to the true ones will exhibit a large correlation value, clearly identified in the left hand side of Figure 3 by a large peak. In contrast, the results on the right hand side of Figure 3 correspond to the case where the search space $\mathcal{S}$ does not contain the true pair of frequency and time values $\left\{f_{*}, \tau_{*}\right\}$ of the received signal. As a result, the local signal replica used in (1) is always incorrectly aligned to the received signal, and in these circumstances, the inner properties of the GPS signal ensure that the resulting signal correlation is negligible. The residual in (1) is then dominated by the additive Gaussian noise introduced by the electronic circuitry of the GPS receiver (i.e. the so-called thermal noise). This is nearly the same situation that would be encountered when the GPS signal is not present, and that is why the results on the right hand side of Figure 3 are said to correspond to the $\mathcal{H}_{0}$ hypothesis (i.e. satellite absent), even though the GPS satellite with PRN 11 being searched is actually present (i.e. it is present but completely misaligned to any of the local signal replicas considered in $\mathcal{S}$ ).
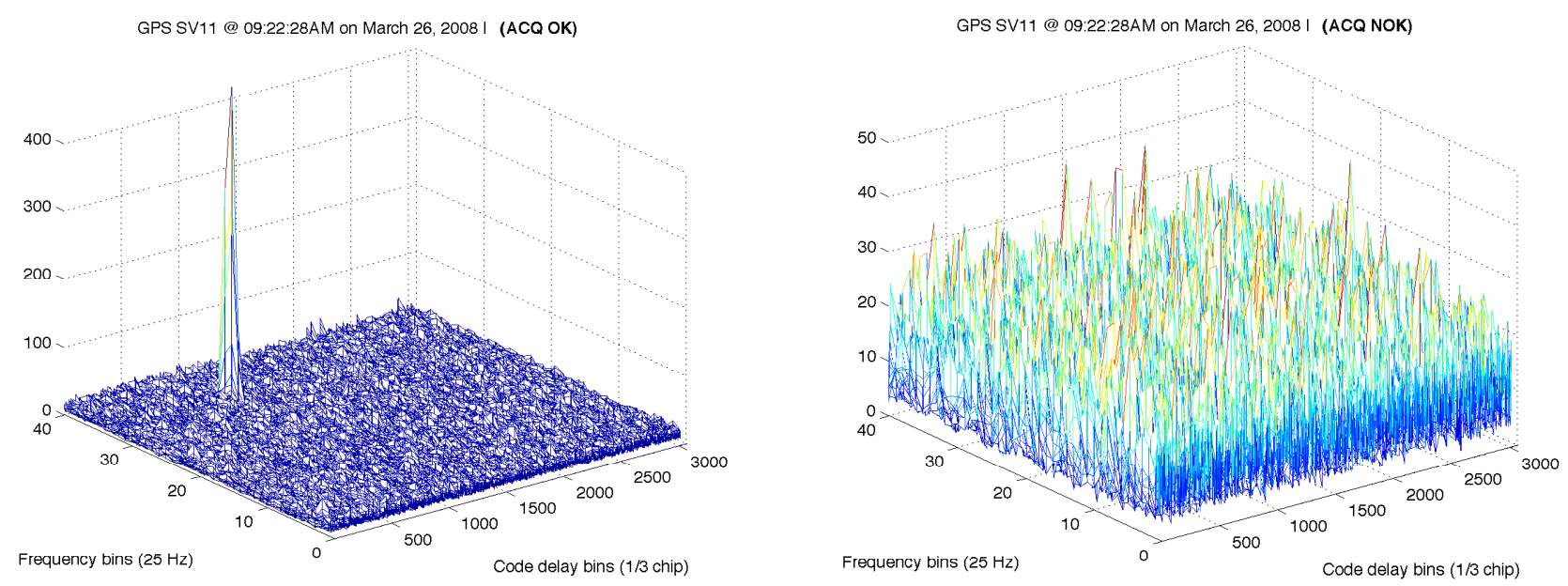

Figure 3. Acquisition matrices for the GPS satellite with PRN code 11, whose signal was recorded on March 26, 2008, at 09:22AM (CET) at Noordwijk, The Netherlands.

Because of the dominance of the thermal noise and the inner properties of the GPS signal, the correlation values in (1) turn out to be Gaussian distributed under $\mathcal{H}_{0}$. That is, $Z_{i}(\hat{f}, \hat{\tau})_{\mid \mathcal{H}_{0}} \sim \mathcal{N}\left(0, \sigma_{w}^{2}\right)$ with $\sigma_{w}^{2}$ the power of the thermal noise. The consequence of this observation is that the entries of the acquisition matrix in (2) become identically distributed $\chi^{2}$ under $\mathcal{H}_{0}$, since

$$
\frac{X\left(\hat{f}_{p}, \hat{\tau}_{q}\right)_{\mid \mathcal{H}_{0}}}{\sigma_{w}^{2}} \sim \chi^{2}\left(2 N_{I}\right)
$$

This statement above can be confirmed in Figure 4, where the empirical density of $X\left(\hat{f}_{p}, \hat{\tau}_{q}\right)_{\mid \mathcal{H}_{0}} / \sigma_{w}^{2}$ is compared to that of $\chi^{2}\left(2 N_{I}\right)$, when using $N_{I}=5$. This example confirms the tight match between the entries of 
the acquisition matrix and the $\chi^{2}$ or Gamma distribution, thus paving the way for the application of the results derived in Section 5 of this manuscript.

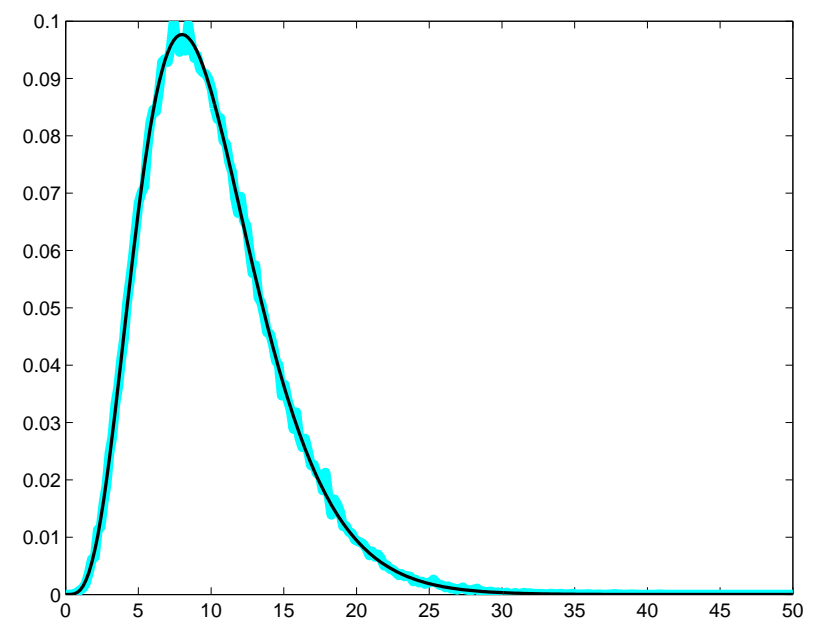

Figure 4. Light blue: empirical distribution for the values on the right hand side of Figure 3 , which correspond to $X\left(\hat{f}_{p}, \hat{\tau}_{q}\right) / \sigma_{w}^{2}$, using $N_{I}=5$ in (2). Black: $\chi^{2}(10)$ distribution.

We will now focus on the distribution of the random variable $M_{n}=\max \left\{X_{1}, \ldots, X_{n}\right\}$ where $X_{i}$ with $i=1, \ldots, n$ follows (6). It has already been discussed in Section 5 that the conventional norming constants $\left\{a_{n}^{\prime}, b_{n}^{\prime}\right\}$ for $M_{n}$, may incur in a significant error when comparing the resulting distribution with the true Gumbel law, i.e. the one using constants $\left\{a_{n}, b_{n}\right\}$ obtained by numerical means. Such a mismatch is clearly visible in Figure 5, where the density of a Gumbel law is plotted and the empirical density of the random variable $M_{n}$ is represented when using the real data from the acquisition matrix on the right hand side of Figure 3 with $n=10^{5}$ entries, using 6000 of these matrices. The density for $Y_{n}^{\prime}=\left(M_{n}-b_{n}^{\prime}\right) / a_{n}^{\prime}$ corresponds to the dotted green line whereas the density for $Y_{n}=\left(M_{n}-b_{n}\right) / a_{n}$ is given by the dashed blue line. In contrast, the new norming constants proposed in this work, namely $\left\{a_{n}^{\prime \prime}, b_{n}^{\prime \prime}\right\}$, led to a random variable $Y_{n}^{\prime \prime}=\left(M_{n}-b_{n}^{\prime \prime}\right) / a_{n}^{\prime \prime}$ whose density, represented by the dashed red line, is almost coincident with the Gumbel law.

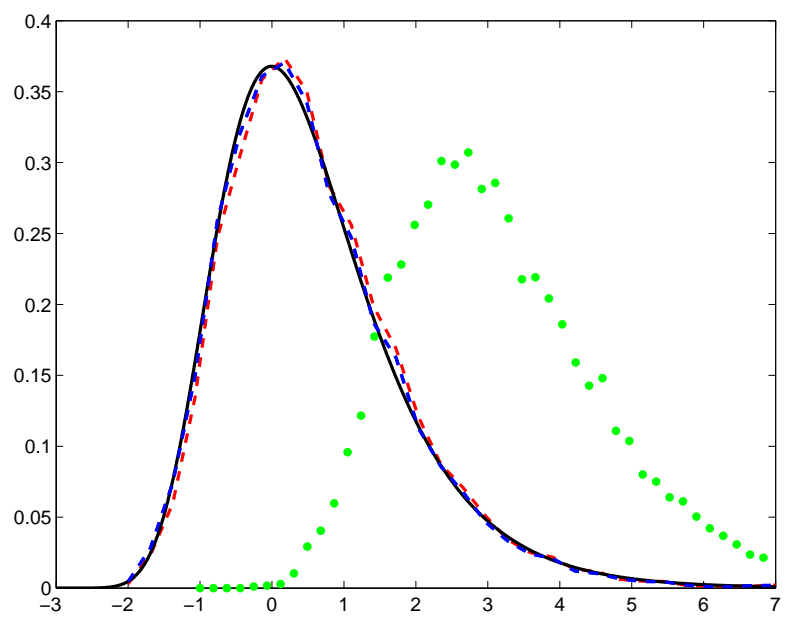

Figure 5. Gumbel density and empirical density of the maxima of $n=10^{5}$ entries of the acquisition matrix constructed with 6000 acquisition matrices without signal. Black solid line: Gumbel density. Thick dashed blue: density of $Y_{n}$. Dashed red: density of $Y_{n}^{\prime \prime}$. Dotted green line: density of $Y_{n}^{\prime}$. 


\section{Online Appendix 2 for TEST article \\ Maxima of Gamma random variables and other Weibull-like distributions and the Lambert $W$ function}

A. Gasull, J. A LóPEZ-S Alcedo, F. UtzeT

\section{On Lambert and Comtet asymptotic expansions and generalizations}

\section{A1. Lambert versus Comtet asymptotic expansions}

Following the notations of Subsection 4.2 of the paper, given $\gamma \neq 0$ denote by $U_{\gamma}(x)$ the (unique) solution of the equation $t^{\gamma} e^{t}=x$ such that $\lim _{x \rightarrow \infty} U_{\gamma}(x)=\infty$. Now, fix $\beta>0$ and consider the equation

$$
t^{\beta} e^{-t}=x
$$

The unique solution of this equation that goes to $\infty$ when $x \rightarrow 0^{+}$can be written in two ways, either by using the negative branch of the Lambert $W$ function, $W_{-1}$, or the function $U_{-\beta}$,

$$
t=-\beta W_{-1}\left(-x^{1 / \beta} / \beta\right)=U_{-\beta}(1 / x) .
$$

From the asymptotic expansion of the negative branch of Lambert $W$ function given in (11) in the paper, the central term of (2) is

$$
\begin{aligned}
t & =-\beta L_{1}\left(x^{1 / \beta} / \beta\right)+\beta L_{2}\left(x^{1 / \beta} / \beta\right)-\beta \sum_{n=1}^{\infty}(-1)^{n+1} \frac{P_{n}\left(L_{2}\left(x^{1 / \beta} / \beta\right)\right)}{L_{1}^{n}\left(x^{1 / \beta} / \beta\right)} \\
& =-L_{1}\left(x / \beta^{\beta}\right)+\beta L_{2}\left(x^{1 / \beta} / \beta\right)-\sum_{n=1}^{\infty}(-\beta)^{n+1} \frac{P_{n}\left(L_{2}\left(x^{1 / \beta} / \beta\right)\right)}{L_{1}^{n}\left(x / \beta^{\beta}\right)}
\end{aligned}
$$

where

$$
L_{1}(x)=\log x \quad \text { and } \quad L_{2}(x)=\log |\log x| .
$$

From Comtet expansion of $U_{\gamma}$ given in (22)of the paper (see Comtet [1]), the term on the right side of (2) is

$$
\begin{aligned}
t & =L_{1}(1 / x)+\beta L_{2}(1 / x)+\sum_{n=1}^{\infty} \beta^{n+1} \frac{P_{n}\left(L_{2}(1 / x)\right)}{L_{1}^{n}(1 / x)} \\
& =-L_{1}(x)+\beta L_{2}(x)-\sum_{n=1}^{\infty}(-\beta)^{n+1} \frac{P_{n}\left(L_{2}(x)\right)}{L_{1}^{n}(x)} .
\end{aligned}
$$

Comparing (3) and (5) we realize that both asymptotic expansions are the same function applied to different points; specifically, define

$$
h(y, z)=-y+\beta z-\sum_{n=1}^{\infty}(-\beta)^{n+1} \frac{P_{n}(z)}{y^{n}} .
$$

Then (3) is the function $h(y, z)$ applied to

$$
y=L_{1}\left(x / \beta^{\beta}\right) \text { and } z=L_{2}\left(x^{1 / \beta} / \beta\right),
$$


whereas (5) is the function $h(y, z)$ applied to $y=L_{1}(x)$ and $z=L_{2}(x)$. The argument of De Bruijn [2, p. 25-27] in this case shows that there exist constants $a$ and $b$ such that if $y>a$ and $0<z / y<b$, then the series in the right hand side of (6) is absolutely convergent and

$$
\left|h(y, z)+y-\beta z+\sum_{n=1}^{N}(-\beta)^{n+1} \frac{P_{n}(z)}{y^{n}}\right| \leq C\left(\frac{z}{y}\right)^{N+1} .
$$

So we deduce

$$
\left|t+L_{1}\left(x / \beta^{\beta}\right)-\beta L_{2}\left(x^{1 / \beta} / \beta\right)+\sum_{n=1}^{N}(-\beta)^{n+1} \frac{P_{n}\left(L_{2}\left(x^{1 / \beta} / \beta\right)\right)}{L_{1}^{n}\left(x / \beta^{\beta}\right)}\right| \leq C\left(\frac{L_{2}\left(x^{1 / \beta} / \beta\right)}{L_{1}\left(x / \beta^{\beta}\right)}\right)^{N+1},
$$

and

$$
\left|t+L_{1}(x)-\beta L_{2}(x)+\sum_{n=1}^{N}(-\beta)^{n+1} \frac{P_{n}\left(L_{2}(x)\right)}{L_{1}^{n}(x)}\right| \leq C\left(\frac{L_{2}(x)}{L_{1}(x)}\right)^{N+1}
$$

Now,

$$
\begin{aligned}
\frac{\frac{L_{2}\left(x^{1 / \beta} / \beta\right)}{L_{1}\left(x / \beta^{\beta}\right)}}{\frac{L_{2}(x)}{L_{1}(x)}} & =\frac{L_{2}\left(x^{1 / \beta} / \beta\right)}{L_{2}(x)} \frac{L_{1}(x)}{L_{1}\left(x / \beta^{\beta}\right)}=\left(1-\frac{\log \beta}{L_{2}(x)}-\frac{\beta \log \beta}{L_{1}(x)}+\cdots\right)\left(1+\frac{\beta \log \beta}{L_{1}(x)}+\cdots\right) \\
& =\left(1-\frac{\log \beta}{L_{2}(x)}+\cdots\right),
\end{aligned}
$$

and, for $x>0$ small enough, (remember $L_{2}(x)=\log (-\log x)<0$, when $x \rightarrow 0^{+}$),

$$
(9) \text { is } \begin{cases}>1, & \text { if } 0<\beta<1 \\ =1, & \text { if } \beta=1 \\ <1, & \text { if } \beta>1\end{cases}
$$

This indicates that

- If $0<\beta<1$ then the finite expansion deduced from (5) seems to produce a more accurate approximation.

- If $\beta>1$, the finite expansion from (3) seems better.

- If $\beta=1$, both expansions are equal.

Intuitively, (3) and (5) are asymptotic expansions when $x \rightarrow 0^{+}$, and, for example, when $\beta>1$, the dominant part of both, $\log (\ldots)$ is applied in (3) to a smaller number, $x / \beta^{\beta}$.

In Table 1 there is a numerical study to illustrate this point. We denote by $t$ the numeric solution of equation (1), by $t_{W}$ the approximation deduced from Lambert expansion (3),

$$
t_{W}=-L_{1}\left(x / \beta^{\beta}\right)+\beta L_{2}\left(x^{1 / \beta} / \beta\right)-\beta^{2} \frac{L_{2}\left(x^{1 / \beta} / \beta\right)}{L_{1}\left(x / \beta^{\beta}\right)},
$$

and by $t_{C}$ the approximation deduced from Comtet expansion (5),

$$
t_{C}=-L_{1}(x)+\beta L_{2}(x)-\beta^{2} \frac{L_{2}(x)}{L_{1}(x)} .
$$




\begin{tabular}{lcccccc}
\hline & & \multicolumn{5}{c}{$\boldsymbol{x}$} \\
\cline { 3 - 6 } & & $10^{-1}$ & $10^{-2}$ & $10^{-3}$ & $10^{-4}$ & $10^{-5}$ \\
\hline $\boldsymbol{\beta}=\mathbf{0 . 5}$ & $\boldsymbol{t}$ & 2.8212 & 5.4533 & 7.9440 & 10.3803 & 12.7871 \\
& $\boldsymbol{t}_{\boldsymbol{W}}$ & 2.8124 & 5.4554 & 7.9464 & 10.3824 & 12.7889 \\
& $\boldsymbol{t}_{\boldsymbol{C}}$ & 2.8102 & 5.4517 & 7.9440 & 10.3808 & 12.7877 \\
\hline $\boldsymbol{\beta}=\mathbf{1}$ & $\boldsymbol{t}$ & 3.5771 & 6.4728 & 9.1180 & 11.6671 & 14.1636 \\
& $\boldsymbol{t}_{\boldsymbol{W}}=\boldsymbol{t}_{\boldsymbol{C}}$ & 3.4988 & 6.4640 & 9.1202 & 11.6717 & 14.1686 \\
\hline $\boldsymbol{\beta}=\mathbf{4}$ & $\boldsymbol{t}$ & 12.3607 & 15.5923 & 18.6005 & 21.4786 & 24.2699 \\
& $\boldsymbol{t}_{\boldsymbol{W}}$ & 11.9175 & 15.3431 & 18.4547 & 21.3922 & 24.2198 \\
& $\boldsymbol{t}_{\boldsymbol{C}}$ & 11.4342 & 16.0199 & 19.1148 & 21.9488 & 24.6826 \\
\hline
\end{tabular}

Table 1. Comparison of the approximations $t_{W}$ and $t_{C}$ given in (10) and (11) with the numeric solution $t$ of equation (1).

\section{A2. Extension of Comtet expansion}

Following the notations of Subsection 5.2.1 of the paper, the solution $U_{\gamma, D}(x)$ of the equation

$$
t^{\gamma} e^{t} D\left(\frac{1}{t}\right)=x
$$

such that $\lim _{x \rightarrow \infty} U_{\gamma, D}(x)=\infty$, has a (formal) asymptotic expansion (see Robin [3])

$$
U_{\gamma, D}(x)=L_{1}(x)+\sum_{n=0}^{\infty} \frac{Q_{n}\left(L_{2}(x)\right)}{L_{1}^{n}(x)}
$$

where the polynomials verify the recurrence relation

$$
Q_{n+1}^{\prime}=-\gamma\left(Q_{n}^{\prime}-n Q_{n}\right), n \geq 0, \quad \text { and } \quad Q_{0}^{\prime}=-\gamma .
$$

Due that this recurrence does not determine the independent term of the polynomials, Salvi [4] considers the generating function of the independent terms of the polynomials, $Q_{0}(0), Q_{1}(0), \ldots$,

$$
\mathcal{G}(s):=\sum_{n=0}^{\infty} Q_{0}(0) s^{n}
$$

and he proves that it satisfies

$$
\mathcal{G}(s)=-\gamma \log (1+s \mathcal{G}(s))-\log D\left(\frac{s}{1+s \mathcal{G}(s)}\right),
$$

which allows to compute the independent terms. Joining (14) and (15) the polynomials $Q_{k}(x)$ can be deduced iteratively. Salvi [4] gives the code of a Maple program to compute recurrently those polynomials. The first two polynomials are

$$
Q_{0}(x)=-\gamma x-\log d_{0} \quad \text { and } \quad Q_{1}(x)=\gamma^{2} x+\gamma \log d_{0}-\frac{d_{1}}{d_{0}}
$$




\section{A3. The secondary branch of the inverse function of $t e^{t} D(1 / t)$}

As we commented in Subsection 5.2.2 of the paper, the equation

$$
t e^{t} D\left(\frac{1}{t}\right)=x
$$

has a unique solution $W_{-1, D}(x)<0$ for $x<0$, such that $\lim _{x \rightarrow 0^{-}} W_{-1, D}(x)=-\infty$. Its asymptotic expansion can be deduced from the very general results of Robin [3] and Salvi [4] commented in Section A2. Indeed, equation (17) is equivalent to

$$
(-t)^{-1} e^{-t} D^{-1}\left(-\frac{1}{-t}\right)=-\frac{1}{x}
$$

where $D^{-1}(t)=1 / D(t)$. Since $\lim _{x \rightarrow 0^{-}}(-1 / x)=\infty$, changing $-t$ by $u$, we have

$$
u^{-1} e^{u} C(u)=-1 / x
$$

where $C(t)=1 / D(-t)$. Hence, with the notations of Section A2, we get $W_{-1, D}(x)=-U_{-1, C}(-1 / x)$. Thus, from (13), the asymptotic expansion of $W_{-1, D}(x)$ is

$$
\begin{aligned}
W_{-1, D}(x) & =-L_{1}(-1 / x)-\sum_{n=0}^{\infty} \frac{R_{n}\left(L_{2}(-1 / x)\right)}{L_{1}^{n}(-1 / x)} \\
& =L_{1}(-x)+\sum_{n=0}^{\infty}(-1)^{n+1} \frac{R_{n}\left(L_{2}(-x)\right)}{L_{1}^{n}(-x)},
\end{aligned}
$$

where the polynomials $R_{n}$ satisfy

$$
R_{n+1}^{\prime}=R_{n}^{\prime}-n R_{n}, n \geq 0, \quad \text { and } \quad R_{0}^{\prime}=1,
$$

and the generating function of the independent terms of the polynomials, $\mathcal{H}(s):=\sum_{n=0}^{\infty} R_{n}(0) s^{n}$, satisfies

$$
\mathcal{H}(s)=\log (1+s \mathcal{H}(s))-\log C\left(\frac{s}{1+s \mathcal{H}(s)}\right)=\log (1+s \mathcal{H}(s))+\log D\left(-\frac{s}{1+s \mathcal{H}(s)}\right) .
$$

Again, from (19) and (20) the polynomials $R_{k}(x)$ can be computed iteratively. The first two polynomials are

$$
R_{0}(x)=x+\log d_{0} \quad \text { and } \quad R_{1}(x)=x+\log d_{0}-\frac{d_{1}}{d_{0}} .
$$

\section{A4 Comparison of two asymptotic expansions}

In a similar way that in Section A1, we are going to compare two asymptotic expansions for the solution of the equation

$$
t^{\beta} e^{-t} A\left(\frac{1}{t}\right)=x
$$

where $\beta>0$ and $A(x)=\sum_{n=0}^{\infty} a_{n} x^{n}$, such that $t \rightarrow \infty$ when $x \rightarrow 0^{+}$. The first way to get the solution is noting that

$$
t=U_{-\beta, A^{-1}}(1 / x)
$$

The (formal) asymptotic expansion is (see Section A2)

$$
t=L_{1}(1 / x)+\sum_{n=0}^{\infty} \frac{Q_{n}\left(L_{2}(1 / x)\right)}{L_{1}^{n}(1 / x)}=-L_{1}(x)-\sum_{n=0}^{\infty}(-1)^{n+1} \frac{Q_{n}\left(L_{2}(x)\right)}{L_{1}^{n}(x)},
$$


where

$$
Q_{n+1}^{\prime}=\beta\left(Q_{n}^{\prime}-n Q_{n}\right), n \geq 0, \quad \text { and } \quad Q_{0}^{\prime}=\beta,
$$

and $\mathcal{G}(s):=\sum_{n=0}^{\infty} Q_{n}(0) s^{n}$, verifies

$$
\mathcal{G}(s)=\beta \log (1+s \mathcal{G}(s))-\log A^{-1}\left(\frac{s}{1+s \mathcal{G}(s)}\right) .
$$

The second asymptotic expansion is deduced from Section A3,

$$
t=-\beta W_{-1, D}\left(-x^{1 / \beta} / \beta\right),
$$

where

$$
D(t)=A^{1 / \beta}\left(-\frac{1}{\beta} t\right),
$$

and $A^{1 / \beta}(t)=(A(t))^{1 / \beta}$. Then, from (18),

$$
\begin{aligned}
t & =-\beta\left(L_{1}\left(x^{1 / \beta} / \beta\right)+\sum_{n=0}^{\infty}(-1)^{n+1} \frac{R_{n}\left(L_{2}\left(x^{1 / \beta} / \beta\right)\right)}{L_{1}^{n}\left(x^{1 / \beta} / \beta\right)}\right) \\
& =-L_{1}\left(x / \beta^{\beta}\right)-\sum_{n=0}^{\infty}(-\beta)^{n+1} \frac{R_{n}\left(L_{2}\left(x^{1 / \beta} / \beta\right)\right)}{L_{1}^{n}\left(x / \beta^{\beta}\right)}
\end{aligned}
$$

where the polynomials $R_{n}$ are determined by (19) and (20). With the notations of Section A3, it follows that $Q_{n}(x)=\beta^{n+1} R_{n}(x)$ : just define the polynomials $S_{n}(x)=\beta^{n+1} R_{n}(x)$ and check that they satisfy (23), and that the corresponding generating function of the independent terms satisfies (24). So, as in Section A1, we deduce that the two asymptotic expansions are the same function applied to different points, and the analysis of Section A1 can be extended to this more general context.

\section{References}

[1] Comtet, L., Inversion de $y^{\alpha} e^{y}$ et $y \log ^{\alpha} y$ au moyen des nombres de Stirling, C. R. Acad. Sc. Paris, Serie A, 270 (1970) 1085-1088.

[2] De Bruijn, N. G., Asymptotic Methods in Analysis, Dover, New York, 1981.

[3] Robin, G., Permanence de relations de récurrence dans certains développements asymptotiques, Publications de l'Institut Mathématique, Nouv. sér., 43 (1988) 17-25.

[4] Salvy, B., Fast computation of some asymptotic functional inverses, J. Symbolic Comput. 17 (1994) 227-236. 